\title{
DEIA White Papers for Planetary 2023 supported by the Cross-AG EDI Working Group
}

Corresponding Authors*: Moses Milazzo ${ }^{1}$, Christina Richey ${ }^{2}$

"As DEIA Working Group Co-Chairs, we request that correspondence about this summary paper be sent to both Corresponding Authors.

${ }^{1}$ Other Orb LLC, (513) 620-4314 (voicemail only), moses@otherorb.net (He, him, his)

2 Jet Propulsion Laboratory, California Institute of Technology, (818) 354-4940, christina.r.richey@jpl.nasa.gov (They, them, theirs)

Co-authors: Julie Rathbun ${ }^{3}$, Edgard G. Rivera-Valintín ${ }^{4}$, Serina Diniega ${ }^{2}$, Jen Piatek ${ }^{5}$, Britney Schmidt $^{6}$, Kathleen Vander Kaaden ${ }^{7}$, Ingrid Daubar ${ }^{8}$, Ross Beyer ${ }^{9}$, Noam Izenberg ${ }^{10}$, Ryan Watkins ${ }^{3}$, Nicolle Zellner ${ }^{11}$, Maggie McAdam ${ }^{12}$, Daniella Scalice ${ }^{12}$, Melissa Kirven-Brooks ${ }^{12}$, Aaron Gronstal $^{12}$, Kat Gardner-Vandy ${ }^{13}$, Zahra Khan ${ }^{14}$, Jacob Richardson ${ }^{15,16}$, Jani Radebaugh ${ }^{17}$, Steve Vance $^{2}$, Matthew S. Tiscareno ${ }^{9}$, Frank Tavares ${ }^{12}$, Mary Beth Wilhelm ${ }^{12}$, Amanda Hendrix ${ }^{3}$, Beck Strauss ${ }^{18,16}$, Abbie Grace ${ }^{19}$

${ }^{3}$ Planetary Science Institute, ${ }^{4}$ Lunar and Planetary Institute (LPI), Universities Space Research Association (USRA), ${ }^{5}$ Central Connecticut State University, ${ }^{6} \mathrm{Georgia}$ Institute of Technology, ${ }^{7}$ Jacobs/NASA Johnson, ${ }^{8}$ Brown University, ${ }^{9}$ SETI Institute, ${ }^{10}$ Johns Hopkins University Applied Physics Laboratory, ${ }^{11}$ Albion College, ${ }^{12}$ NASA Ames Research Center, ${ }^{13}$ Oklahoma State University, ${ }^{14}$ Independent Research, ${ }^{15}$ University of Maryland College Park, ${ }^{16}$ NASA Goddard Space Flight Center, ${ }^{17}$ Brigham Young University, ${ }^{18} \mathrm{NIST},{ }^{19}$ University of Tasmania

The EDI Working Group steering committee members:

- CAPTEM: Justin Filiberto (LPI) \& Liz Rampe (NASA Johnson)

- LEAG: Ryan Watkins (PSI) \& Kelsey Young (NASA Goddard)

- MAPSIT: Jani Radebaugh (BYU) \& Alex Patthoff (PSI)

- MEPAG: Justin Filiberto (LPI) \& TBD

- OPAG: Linda Spilker (JPL) \& Kunio Sayanagi (Hampton University)

- SBAG: Jennifer Scully (JPL) \& Jessie Dotson (NASA Ames)

- VExAG: Noam Izenberg (APL) \& Emilie Royer (PSI)

- MExAG: Kathleen Vander Kaaden (Jacobs/NASA Johnson) \& Gangkai Poh(NASA GSFC)

- DPS PCCS: Ed Rivera-Valentin (LPI), Julie Rathbun (PSI), \& Serina Diniega (JPL)

- Special: Janet Vertesi (Princeton) 
Diversity, Equity, Inclusion and Accessibility (DEIA) ${ }^{1}$ efforts within the Planetary Science community have become a focus point of several NASA Assessment Groups (AGs) in recent years. The DEIA Working Group (WG) was formed by the AGs and its steering committee consists of members of each AG steering committee and several affinity groups, and is charged with creating and disseminating an organized structure to provide recommendations, resources, and findings associated with DEIA issues to the larger AG Steering Committees and to the greater planetary science community. The WG is open to all interested planetary scientists and currently has more than 50 members.

The WG has gathered community input in the form of a series of Professional White Papers in preparation for the Planetary Science Decadal Survey. In this overview paper, we summarize several of the papers that have been organized and submitted through efforts of community members associated with the WG. Note that we are combining these ideas here to make it easier for the decadal survey panelists to find papers on specific topics. We use the summary language of the original papers and we endorse all of the information and recommendations in those papers; the ideas did not originate with us and credit should be given to the authors of the individual white papers. ${ }^{2}$ There are likely other papers that have been submitted by members of the community who may not be associated with the WG; many of those have been co-authored, co-signed, or are supported by our members. There are papers that were put together after our deadline for contributions to be included in this document, so we do not summarize every paper that's been submitted. We fully support their findings and recommendations as well. We strongly recommend that the Decadal Survey Panel read each of the submitted papers and use those papers as primary citations rather than citing this summary paper.

White Papers Summaries:

\section{Who is Missing in Planetary Science?: A demographic study of the planetary science workforce, Rivera-Valentín, et. al.}

In this white paper, we conducted a demographic analysis, focused on race, ethnicity, and the representation of women by self-identification, based on the American Astronomical Society's (AAS) Division of Planetary Sciences (DPS) 2011 and 2020 workforce surveys, along with national sources, to identify trends in underrepresentation. Currently, women are underrepresented by $28.0 \% \pm 5.2 \%$, American Indian / Alaskan Native by $42.3 \% \pm 37.6 \%$, Latinx / Hispanics by $76.3 \% \pm 5.7 \%$, and Black / African Americans by $91.6 \% \pm 4.0 \%$ with respect to the National Civilian Labor Force (NCLF). Comparison of the 2011 and 2020 workforce surveys finds that while women and Latinx / Hispanics have seen increases in

\footnotetext{
${ }^{1}$ Improving equity promotes justice, impartiality, and fairness within the procedures, processes and distribution of resources by institutions and systems. Tackling equity issues requires an understanding of the root causes of disparities within our society. Diversity refers to a broad representation of a community's demographic mix, considering elements of human difference focusing on racial and ethnic groups, sexual orientation, gender identity, abilities, religion, age and perspectives arising from different backgrounds. Inclusion refers to the degree in which diverse individuals are able to participate fully in the decision-making processes within an organization or group. While a truly "inclusive" group is necessarily diverse, a "diverse" group may or may not be "inclusive." Accessibility refers to the aspects of an environment that influence peoples' abilities to function within that environment.

${ }^{2} \mathrm{~A}$ list of all DEIA Working Group-related white papers is available at https://docs.google.com/spreadsheets/d/1t5hpGpVIWokwaymDITyMaYAVWJ4viFTsXDJVwTQb9gQ/edit\#gid=0
} 
representation while Black / African Americans have seen no growth in the past 9 years. Additionally, comparing the current population of student researchers in planetary science to non-student researchers, we find that women and American Indian / Alaskan Natives have significant improvements in their representation, rising to parity with respect to the NCLF; however, Latinx / Hispanics and Black / African American planetary scientists remain significantly underrepresented.

\section{Who is missing in Planetary Science?: Strategic Recommendations to Improve the Diversity of the Field, Rathbun, et al.}

Racial and ethnic minorities (particularly those of African American, Latinx, and Native American background) are the most underrepresented groups in planetary science. Comparisons of the numbers of planetary scientists to the US population show that these groups are more underrepresented than white or Asian scientists by a factor of $\sim 10$. We share social science results that could help explain the disparity. We make recommendations that can be implemented to improve the climate for members of underrepresented groups in planetary science.

\section{Enabling the Planetary Workforce to do the best science by funding work that is a service to the Profession by Rathbun, et al.}

Service work is required to keep science moving and improving. Being involved in the decadal survey process is a service to the profession. Doing work to understand the state of the profession is a service to the profession. Here, we give ways to enable these service jobs to be equitably distributed, valued, and funded in our community.

\section{Ensuring a safe and equitable workspace: the importance and feasibility of a Code of Conduct, Diniega, et al.}

As our field aims to become more inclusive, we need updated community and social practices to ensure that everyone (especially those with less power) have safe and equitable workspaces. This white paper will primarily focus on the "Code of Conduct" as a policy/process by which guidance is provided for interactions between team/group members or conference attendees. Generally, these statements are written with an aim to foster a more inclusive and accessible environment by protecting the physical, mental, and emotional safety of all participants. We also will discuss a few other policies with regards to paper authorship and field/travel planning that are important for improved diversity, equity, inclusivity, and accessibility.

5. Breaking Down Barriers: Accessibility in Planetary Science, Piatek, et al. Planetary science relies on a diversity of disciplines to explore the solar system, but we are missing diversity in our community due to barriers erected by inaccessible workplaces and negative perceptions about required accommodations. We address why accessibility should matter to planetary scientists and how to address barriers within our community. This is an old challenge addressed by other groups dedicated to increasing accessibility across the sciences. Our broad recommendation is the adoption of policies from appropriate advocacy groups, including the Working Group on Accessibility and Disability of the American Astronomical Society and the International Association for Geoscience Diversity.

\section{Ensuring Inclusivity in the 2023 Planetary Science and Astrobiology Decadal Survey, Rathbun, et al.}

Inclusivity is a difficult, but necessary goal, particularly of a process that aims to represent the consensus of a large group. Here, we give recommendations to address 2 main questions: (1) How can we ensure that the voices of the most marginalized in Planetary science are represented in the current Decadal Survey Process? (2) How can we ensure that DEIA and the state of the 
profession are given the consideration they require in order to make recommendations that will improve the inclusivity of the Planetary Science and Astrobiology communities?

7. Lessons learned on IDEA from the Astro2020 Decadal Survey, Richey, et al. The Decadal Survey on Astronomy and Astrophysics 2020 (Astro2020) is currently in the drafting phase of the study, which will provide recommendations for the next decade of astrophysics research. Among the tasks requested was to assess the state of the profession. Astro2020 received 294 White Papers concerning Activities, Projects, and the State of the Profession. Summarized here are the recommendations from several of the DEIA (Diversity, Equity, Inclusion, and Accessibility) White Papers that could also be applicable and critical to the Planetary Science and Astrobiology Decadal Survey (2023-2032).

\section{Diversity in action: Solutions for a more diverse and inclusive decade of planetary science and astrobiology, Britney Schmidt, et al.}

There are short-term and long-term changes to NASA programs, institutional structures, and incentives that have the potential to markedly improve diversity and equal opportunity. We have made ten recommendations that range from easy to implement to those that require research-backed approaches and infusion of new resources. We have structured the order of these programs based on a rough estimate of the cumulative positive impact these changes may have for underrepresented groups, but all of these changes positively impact the entire PS\&A community. For example, eliminating bias community-wide through training removes barriers to entry for diverse groups, while also improving the broader community. Infusion of resources into traditionally underrepresented groups takes dedicated effort, but has the benefit of increasing pipelines of diverse people into the community, which has been shown to improve the quality of work as well. We emphasize that these are meant as starting points for a discussion that must be undertaken as part of a much broader commitment to changing the way the scientific community is built and supported. It will take the work of diverse minds and strong personal and institutional commitments to effect lasting change.

\section{Creating Inclusive, Supportive, and Safe Environments in Planetary Science for Members of the LGBTQ+ Community, Vander Kaaden, et al.}

Although a recent survey of planetary scientists shows that LGBTQ+ representation has grown in this field from 3\% in 1970 to $12 \%$ in the last 5 years, many members of this community are still marginalized, excluded, and unsafe in planetary science. It is critical that we begin to foster an interdisciplinary, diverse, equitable, inclusive, and accessible environment over the next decade, especially for members of the LGBTQ+ community. We recommend numerous actionable items that can be taken to make planetary science workplaces, professional societies, professional conferences, and universities more inclusive, supportive, and safe environments for members of the LGBTQ+ community.

\section{Recommendations from the CSWA Survey on Workplace Climate, Richey, et al.} In planetary science, the dominant lived experience \& cultural constructions (including power \& influence) of the field are white, cisgender, male, \& straight. This paper looks at the results from the CSWA Workplace Climate Survey, an internet-based survey of the workplace experiences of 474 astronomers \& planetary scientists between $2011 \& 2015$. We will also present the recommendations from our peer reviewed papers, which largely reference the American Physical Society LGBT+ best practices report [Ackerman et al., 2018], \& the National Academies of Science, Engineering, \& Medicine (2018) report on sexual harassment. With this, we seek not 
only to highlight a problem but also to provide a path toward a more inclusive \& equitable scientific community.

\section{Extended Missions in Planetary Science: Impacts to Science and the Workforce, Ingrid Daubar and Beyer, et al.}

Extended missions contribute tremendous demonstrated value. The return in science productivity more than justifies the relatively small expense of extending functioning missions. We ask the Decadal Survey to explicitly highlight the value of extended missions to the planetary community, both in terms of scientific achievements and as an important career pipeline. We encourage an evaluation and response to the recommendations in the 2016 National Academies Report "Extending Science". Additionally, we recommend several actions that would improve clarity, attempt to do better at providing robust and stable funding, and improve participation in and inclusivity of missions.

\section{Planetary and Astrobiology Blank Papers: Science White Papers Cancelled or Downscaled Due to Direct Impact of COVID-19 and National-scale Civil Action, Izenberg, et al.}

A significant number of community members intended to submit white papers but were unable to do so due to effects of COVID-19 and disruptions due to recent national civil action. This list informs the Decadal Survey of some of those missing voices, providing a resource for the Committees to fill gaps caused by barriers to participation.

\section{Professional development in the next decade: Supporting opportunities in all career} paths and life events, Watkins, Zellner, and McAdam, et al.

Planetary Science and Astrobiology need more than numerical diversity to create and sustain a thriving scientific discipline and community; they also need scientists from a variety of backgrounds, institutions, and family situations. Support is particularly needed for people who parent and/or care for others, people who breastfeed, people who work at Primarily Undergraduate Institutions (PUIs) and Minority Serving Institutions (MSIs), and people who choose career paths that do not include research at large universities or NASA centers. Additionally, funding professional development that includes proposal writing, proposal review, manuscript review, as well as pro-social skills such as bystander intervention, could substantially support or increase continued participation in the field. By providing supportive work and conference environments, as well as opportunities for scientists to develop skills and collaborations that will further their careers, the fields of Planetary Science and Astrobiology will become more just and equitable.

14. The Preventing Harassment in Science Workshop: Summary and Recommendations of Best Practices for Planetary Science and Astrobiology, Bennett, et al.

The Preventing Harassment in Science: Building a Community of Practice for Meaningful Change workshop took place in June of 2020. This highly successful, NASA-funded workshop brought leaders of anti-harassment efforts together to share ideas and discuss best practice methods to reduce harassment in the scientific workplace. In this white paper, we describe the workshop and summarize the best practices for reducing harassment that were discussed. We include a list of recommendations that can be used to take steps towards reducing harassment in the planetary science and astrobiology community.

\section{Power and Responsibility, Scalice, Kirven-Brooks and Gronstal, et al.}

In addition to implementing new EDI policies and practices within their own walls, agencies have the opportunity to recognize and claim the power they hold in their working relationships, 
and utilize that power to exert influence on how those with whom they do business and affiliate conduct themselves with respect to EDI. Agencies have an inherent stake in the EDI health of the institutions to which they transmit funds and with which they cultivate long-term working relationships. Researchers funded by agencies like NASA have a strong role to play and a vital stake in how their institutions treat EDI. Agencies can provide motivation to make needed changes at an institution via its solicitation/proposal review/award processes.

\section{Relationships First and Always: A Guide to Collaborations with Indigenous \\ Communities, Gardner-Vandy and Scalice, et al.}

In this paper, we outline recommendations for working with Indigenous Communities based on the knowledge that long-term relationship-building with these communities is the foundation upon which educational programs, research collaborations, and other initiatives must be co-created. The paper defines a series of best practices in approach and process for establishing and maintaining successful collaborations with Indigenous communities which have application across many types of efforts. Implementing these practices will have lasting impacts on EDI policies of institutions including universities, professional societies, educational organizations, and agencies, on the STEM workforce broadly, and for Indigenous youth and communities to come to identify with and support the pursuit of STEM as a career.

\section{Military Work by Space Exploration Organizations: A Barrier to Inclusion and Safe}

Workspaces for Marginalized Communities, Khan, et al

Space science labs are involved with military industrial complex (MIC) organizations working on technologies such as weapons, surveillance aircraft and spacecraft, and machine learning and artificial intelligence for military use. While many members of the space science community have positive associations with the United States military, police and intelligence agencies, these agencies' extensive history of targeting BIPOC abroad and at home, though concerning for all, may have a higher negative impact on marginalized members of our profession and allies working in spaces with ties to these agencies by posing ethical dilemmas in the workplace. These impacts could affect employee engagement and retention rates, and whether or not students enter the space science field in the first place. We recommend that studies be conducted to quantify and understand the extent of the impact of space science's ties to the MIC on EDI in space science and further that projects pursue full transparency in their ties to organizations that participate in the MIC.

18. Building safer and more inclusive field science, Richardson, et al.

Field expeditions in support of planetary science is important to advance our understanding of planetary processes and enhance the science community through training and close, often interdisciplinary collaborative efforts. Still, field work faces unique safety risks and barriers to entry, due to the physical nature of the field but also from team behavior and sometimes inhospitable communities near common field sites. Field teams need to be resilient to field site hazards and self-supportive to improve safety and accessibility. NASA should call on proposing teams to develop robust safety plans and should develop a code of conduct for field research funded by NASA. Field teams should equally value physical safety training (e.g. First Aid and CPR) and social safety training (e.g., Bystander Awareness Training).

19. The Value of Dual Anonymous System for Reducing Bias in Reviews of Planetary Research and Analysis Proposals and Scientific Papers, Radebaugh, et al.

Reviewer anonymous paper and proposal review systems have been in place for many decades. Dual anonymous review systems, in which neither the submitting party nor the reviewing party 
are known to each other, have the potential to improve the success rate of scientific proposal and paper acceptance with underrepresented or marginalized groups. Often conscious or unconscious bias can occur on the behalf of the reviewer, in which a person's capabilities are inferred based on their gender, ethnicity, age, affiliation or relative newness to the field. Concealing the identity of the proposal or paper author removes the potential for unconscious bias and allows for the work to be evaluated on its own merit. The Space Science Telescope Institute (STScI) observed significant improvements in the amount of telescope time awarded to female scientists under their dual anonymous review system (Johnson et al. 2020).

20. Addressing Mental Health in Planetary Science, Vance, et al.

As NASA strives to be more inclusive by confronting institutional racism, gender inequality, and sexual harassment, and recognizing sexual diversity, it must also work to address a compounding crisis of mental health. We summarize the available evidence for a mental health crisis among academics. We describe how this problem intersects with and amplifies problems of equity, diversity, and inclusion.

\section{Planetary Nomenclature and Indigenous Communities, Tiscareno, et al.}

In recent decades, planetary nomenclature has expanded from drawing almost exclusively from European cultures to become more culturally diverse. However, Indigenous communities have generally not been included in decisions to use their cultural property for planetary nomenclature. Actively including Indigenous voices in naming decisions ensures that their cultures are accurately and respectfully represented and recognizes their sovereignty over their own culture. The planetary community and NASA should build strong co-creative relationships with Indigenous communities, eventually resulting in procedures for responsible use of cultural property in planetary nomenclature.

\section{The Growing Digital Divide and its Negative Impacts on NASA's Future Workforce, Milazzo et al.}

COVID-19 has exposed and exacerbated the digital divide and digital inequalities especially for Black, Indigenous and People of Color (BIPOC); low income; urban; and rural families and students. This includes families and students with a wide variety of mental health issues and disabilities that can be negatively impacted by online educational models. It is well-understood that early access to STEM and technology in general has a positive impact on student performance, degree completion, and science and graduate school enrollment (11). This growing digital divide and its sudden increase caused by COVID-19 will have negative impacts on NASA's workforce in 10 years or fewer, when these students are (or are not) graduating from high school or with undergraduate degrees and are making decisions about undergraduate and graduate school and majors.

\section{Ethical Exploration and the Role of Planetary Protection in Disrupting Colonial Practices, Tavares and Wilhelm, et al.}

We recommend that the planetary science and space exploration community engage in a robust discussion on the ethics of how future crewed and uncrewed missions to the Moon and Mars will interact with those planetary environments, with a special emphasis on how such missions can resist colonial structures. Such discussions must be rooted in the historical context of the violent colonialism in the Americas and across the globe that has accompanied exploration of Earth.

24. Results of the 2020 Planetary Workforce Survey conducted by the DPS, Hendrix, et al. Understanding the changing demographics and needs of the planetary science community is an important part of effectively serving the community. In this paper we report on initial results of a 
planetary workforce survey conducted by the DPS during 2020. Findings include that the majority of scientists use NASA grants to fund their research activities and that few planetary scientists have proposed a mission as a PI with white men overrepresented (compared to their representation in the community) on mission proposals as either PI or Co-I.

\section{A Call to Planetary 2023 Panels to Implement Actionable Recommendations from Recent National IDEA Studies, Richey, et al.}

While demographic representation in the community is the focus of several other white papers submitted to Planetary2023, understanding the workplace environments beyond diversity is needed to assess both inclusion and accessibility. Inclusion, Diversity, Equity, and Accessibility (IDEA, also referred to as DEIA) as they apply to scientific disciplines such as planetary science, have been intensely studied by professional social scientists for decades. Major reports have been conducted by professional societies and the National Academy on topics that are highly applicable to Task \#9 of Planetary2023. Here, we summarize five major reports and studies and highlight the findings that would be critical to push forward in the planetary science community. 26. Nonbinary Systems: Looking to the future of gender equity in planetary science, Strauss, et al.

Although there is broad interest in addressing gender equity within planetary science, many studies of this topic have been performed by professional space scientists who are unfamiliar with research in fields such as gender studies and sociology; these studies tend to adopt a normative view of gender as a binary choice of 'male' or 'female' that leaves planetary scientists whose genders do not fit within that model out of such research entirely. These approaches are harmful to people of marginalized genders, especially those who live at the intersections of multiple axes of marginalization such as race, disability, and socioeconomic status. In order for the planetary science community to best serve its marginalized members as we move into the next decade, we aim to address the future of gender equity in planetary science by recommending better survey practices and institutional policies based on a more complex approach to gender.

27. Promoting the 'A' in SPACE: 'Arts' run the places STEM takes us, Grace et al. Planetary science and other space-focused activities are supported by increasing efforts to promote STEM subjects and skills, but it is arts professions such as education, governance, commerce, workforce relations, international cooperation, communications/engagement, law and ethics evaluations that support our work and are becoming space-specialist fields in their own right. Equitable participation in the space realm depends not only on looking at individuals' experiences, but empowering 'non-science' professionals to participate without having to leave their field of expertise - either by bringing their perspective into traditional space programs or by creating new roles and collaboration opportunities for space-educated arts professionals. As space becomes increasingly crowded and contested, it is becoming more urgent than ever to genuinely promote and resource these new crossovers of STEM and arts. 